\title{
Prevalence of temporomandibular disorder in children and adolescents with juvenile idiopathic arthritis - a Norwegian cross- sectional multicentre study
}

J. Fischer ${ }^{1 *}$ D, M. S. Skeie ${ }^{1,2}$, K. Rosendahl ${ }^{3,4}$, K. Tylleskär ${ }^{5}$, S. Lie' ${ }^{1}$, X.-Q. Shi ${ }^{1,6}$, E. Grut Gil ${ }^{1}$, L. Cetrelli' ${ }^{2}$ J. Halbig ${ }^{7}$, L. von Wangenheim Marti ${ }^{1}$, M. Rygg ${ }^{8,9}$, P. Frid ${ }^{7,10,11}$, P. Stoustrup ${ }^{12}$ and A. Rosèn ${ }^{1,13}$

\begin{abstract}
Background: Children and adolescents with juvenile idiopathic arthritis (JIA) may suffer pain from temporomandibular disorder (TMD). Still, routines for the assessment of temporomandibular joint (TMJ) pain in health and dental care are lacking. The aims of this study were to examine the prevalence of TMD in children and adolescents with JIA compared to their healthy peers and to investigate potential associations between JIA and TMD.

Methods: This comparative cross-sectional study is part of a longitudinal multicentre study performed during 20152020, including 228 children and adolescents aged 4-16years with a diagnosis of JIA according to the ILAR criteria. This particular substudy draws on a subset of data from the first study visit, including assessments of TMD as part of a broader oral health examination. Children and adolescents with JIA were matched with healthy controls according to gender, age, and centre site. Five calibrated examiners performed the clinical oral examinations according to a standardised protocol, including shortened versions of the diagnostic criteria for TMD (DC/TMD) and the TMJaw Recommendations for Clinical TMJ Assessment in Patients Diagnosed with JIA. Symptoms were recorded and followed by a clinical examination assessing the masticatory muscles and TMJs.

Results: In our cohort of 221 participants with JIA and 221 healthy controls, 88 (39.8\%) participants with JIA and 25 (11.3\%) healthy controls presented with TMD based on symptoms and clinical signs. Painful TMD during the last 30 days was reported in 59 (26.7\%) participants with JIA vs. $10(5.0 \%)$ of the healthy controls $(p<0.001)$. Vertical unassisted jaw movement was lower in participants with JIA than in controls, with means of $46.2 \mathrm{~mm}$ vs. $49.0 \mathrm{~mm}$, respectively $(p<0.001)$. Among participants with JIA, a higher proportion of those using synthetic disease-modifying antirheumatic-drugs and biologic disease-modifying antirheumatic-drugs presented with painful masticatory muscles and TMJs at palpation.

(Continued on next page)
\end{abstract}

* Correspondence: Johannes.Fischer@uib.no

'Department of Clinical Dentistry, The Faculty of Medicine, University of Bergen, Årstadveien 19, N-5009 Bergen, Norway

Full list of author information is available at the end of the article

C C The Author(s). 2020 Open Access This article is licensed under a Creative Commons Attribution 4.0 International License, which permits use, sharing, adaptation, distribution and reproduction in any medium or format, as long as you give appropriate credit to the original author(s) and the source, provide a link to the Creative Commons licence, and indicate if changes were made. The images or other third party material in this article are included in the article's Creative Commons licence, unless indicated otherwise in a credit line to the material. If material is not included in the article's Creative Commons licence and your intended use is not permitted by statutory regulation or exceeds the permitted use, you will need to obtain permission directly from the copyright holder. To view a copy of this licence, visit http://creativecommons.org/licenses/by/4.0/. The Creative Commons Public Domain Dedication waiver (http://creativecommons.org/publicdomain/zero/1.0/) applies to the data made available in this article, unless otherwise stated in a credit line to the data. 
(Continued from previous page)

Conclusion: Symptoms and clinical signs of TMD were seen in approximately half of the JIA patients compared to about one fourth of their healthy peers. Painful palpation to masticatory muscles and decreased vertical unassisted jaw movement were more frequent in participants with JIA than among healthy controls and should be part of both medical and dental routine examinations in patients with JIA.

Keywords: Juvenile idiopathic arthritis, Temporomandibular joint arthritis, Temporomandibular disorder, Temporomandibular joint disease, Children and adolescents

\section{Background}

Juvenile idiopathic arthritis (JIA) is currently the most common chronic rheumatic disease in children and adolescents [1, 2]. The International League of Associations of Rheumatology (ILAR) defines JIA as arthritis of unknown aetiology, starting before the age of 16 years with a duration of at least 6 weeks [3]. It encompasses seven categories, including systemic arthritis, oligoarthritis (persistent or extended), rheumatoid factor negative polyarthritis, rheumatoid factor positive polyarthritis, psoriatic arthritis, and enthesitis-related arthritis with different, though overlapping, characteristics. Cases that fit none or more than one of these categories are defined as undifferentiated arthritis. The burden of JIA is characterised by short and long-term functional disability and pain. Common features at presentation are morning stiffness, swelling of one or more joints, functional disturbances, and sometimes pain. The reported prevalence is around 1-2 cases per 1000 children, with girls more frequently affected than boys [1, 2].

Temporomandibular disorder (TMD), known as an umbrella or collective term for muscle pain and jaw dysfunction, covers a heterogeneous group of conditions [4]. TMD is associated with various clinical signs and symptoms involving the masticatory muscles, teeth, tongue, temporomandibular joint (TMJ), and/or their supportive tissues [5-7]. Changes in motor behaviour caused by musculoskeletal pain and pain-related movement disorders reflect sustained pain perception. In two recent studies from Western Norway $[8,9]$, the prevalence of painful TMD among otherwise healthy adolescents was reported to be around $7 \%$ based on selfreported pain screening questionnaires adopted by Nilsson and colleagues [10]. In the study by Graue and colleagues [9], the prevalence of TMD was $11.9 \%$ when using the Diagnostic Criteria for Temporomandibular Disorders (DC/TMD). In all three studies, females were more frequently affected than males.

In children and adolescents with JIA, the reported figures are substantially higher. Previous studies of children and adolescents with JIA reported a broad spectrum of TMD prevalence ranging between 39 and $87 \%$ [11-13], depending on the study designs and the sample size. Therefore, it is a need to reinforce the evidence with a relatively high number of samples and give insights to the effects of medication in JIA participants when they are examined by palpation of masticatory muscles and TMJ.

Previous studies revealed that children and adolescents, irrespective of their JIA category, are prone to develop TMJ arthritis [11, 14]. Also, younger children with JIA might suffer pain from TMJs caused by inflammation and/or destructive changes, by muscular tensions from the surrounding muscles as a component of TMD, or by a combination of the two [12]. Symptoms indicating TMJ arthritis include decreased mouth opening and/or ear ache and pain during eating, chewing, or yawning [15-17]. At present, there are no precise clinical or imaging markers for active TMJ arthritis [18, 19]. As for TMJ involvement, several studies have shown that even significant deformities may be undiagnosed due to a lack of symptoms or clinical findings [16, 20-22]. In younger children, the clinical assessment of painful TMD symptoms might be biased by indirect input from their parents.

The aims of the present study were to examine the prevalence of TMD in children and adolescents with JIA compared to their healthy peers and to investigate potential associations between JIA and TMD.

\section{Methods \\ Study design and participants}

This cross-sectional study was part of a longitudinal multicentre study, the NorJIA study, performed during 2015-2020 and including 228 children and adolescents. Inclusion criteria were a diagnosis of JIA according to the ILAR [3] and age 4-16 years. In the exclusion criteria, absent of written informed consent or major medical comorbidities such as congenital facial anomalies, skeletal dysplasia or malignancies were excluded.

This particular substudy (2015-2018), using a matched comparative cross-sectional design, drew on a subset of data from the first study visit, including assessments of TMD as part of a broader oral health examination. Children and adolescents were matched (1:1) with healthy controls according to gender, age, and centre site. The healthy controls were recruited from seven different Public Dental Service clinics representing both rural and urban areas in the western, middle, and northern parts 
of Norway. The sample size estimate was based on a Swedish study reporting a TMD prevalence of $26 \%$ in children with JIA [23], and a sample size of 296 was required for a precision of $5 \%$ with a $95 \%$ confidence interval.

\section{Data collection}

At the study visits, children and adolescents with JIA were examined by experienced paediatric rheumatologists at Haukeland University Hospital in Bergen, University Hospital of North Norway in Tromsø, and St. Olavs University Hospital in Trondheim. Registered data included background characteristics in terms of age at disease onset, disease category, disease status on the day of the examination, a thorough joint examination, blood tests, and validated measures for patient-reported disability, general body pain, and health assessments. Furthermore, the applied dose was according to the international recommendations, while duration varied significantly, with or without combination with other medication. However, detailed drug history concerning duration and doses was not available in the study database. Both children and adolescents with JIA and controls underwent a thorough clinical oral examination performed by experienced dentists, including a TMD assessment.

\section{TMD screening and assessment}

The assessment procedures were standardised and were based on two shortened versions of the diagnostic tools "Axis I Clinical Examination for DC/TMD" [20] and the self-assessment questionnaire "TMJaw Recommendations for Clinical TMJ Assessment in Patients Diagnosed with JIA" [21]. The latter was used to enhance the operational specification of DC/TMD due to the fact that the DC/TMD tool alone is reported to show weak validity for TMJ assessment, e.g. disc displacement diagnosis (low sensitivity) and degenerative joint disease diagnosis (low sensitivity and specificity) [20].

Prior to and during the study period, calibration sessions for the five participating oral examiners were performed, including four calibration exercises according to procedures previously described by our research team [22]. Further details on the calibration results are presented in Supplementary Tables S1 and S2.

\section{Variables and outcomes}

The demographic variables were age, gender, JIA categories, and medication status. The subjective symptom outcomes were TMD pain in the last 30 days (n, \%) reported by the participants or the parents. The examining dentists also registered how many of the individuals expressed pain during jaw movement in the clinical examination (n, \%). The clinical outcomes included vertical and lateral unassisted jaw movements $(\mathrm{mm})$, pain upon palpation of the masticatory muscles and the TMJ (n), and if the TMJ disc was clicking in a painful manner (n).

\section{Statistical methods}

Two-way mixed intraclass correlation coefficient (ICC) and percent agreement were used for calibration measurements. Differences between groups were tested using Chi-square statistics or a two-sample t-test as appropriate. All statistical tests were performed using SPSS version 25 (IBM, Chicago, IL). The level of statistical significance was set at $5 \%(p \leq 0.05)$.

\section{Ethical considerations}

The study was approved by the regional ethics committee (2012/542/REK vest). Written informed consents were obtained from all parents and/or participants as appropriate. The study was registered at ClinicalTrials.gov (No: NCT03904459).

\section{Results}

A total of 360 children and adolescents with JIA were eligible for the main study, of whom 228 accepted the invitation to participate, yielding a response rate of $63.3 \%$. The acceptance rate for healthy controls was $224 /$ 294 (76.2\%). The mean age for participants with JIA and healthy controls was 12.0 years (SD 3.17 and 3.21, respectively) $(p=0.98)$, and the mean age of the 228 participants with JIA was higher than for the 132 eligible patients that did not participate at 12.0 years vs. 10.5 years (SD 3.16 and 3.5 , respectively) $(p<0.001)$. The proportion of girls with JIA was also higher than among the 132 patients not participating $(59.2 \%$ vs. $58.3 \%, p=$ 0.027). Among the 228 participating children with JIA, 224 underwent an oral examination and 221 underwent the TMD assessment and were thus included in the present substudy (Fig. 1).

Of the 221 children with JIA, 132 were girls (59.7\%), the median age at disease onset was 6.1 years (IQR 8.1, 2.310.4 ), the median age at the study visit by paediatric rheumatologists at the hospital was 12.7 years (IQR (5.3, 9.414.7), and the median disease duration was 4.6 years (IQR 5.7, 2.6-8.3) (Table 1). Oligoarticular JIA was the most common category and was seen in 98 of 221 patients (44.3\%) with 77 having persistent oligoarticular disease and 21 having extended disease. In total, 146 of the 221 patients (66.1\%) had on-going medication with synthetic diseasemodifying antirheumatic-drugs (sDMARDs) and/or biologic disease-modifying antirheumatic-drugs (bDMARDs).

\section{Clinical oral examination}

Taking into consideration that self-reported pain is a combination of parent-reported and participant-reported 

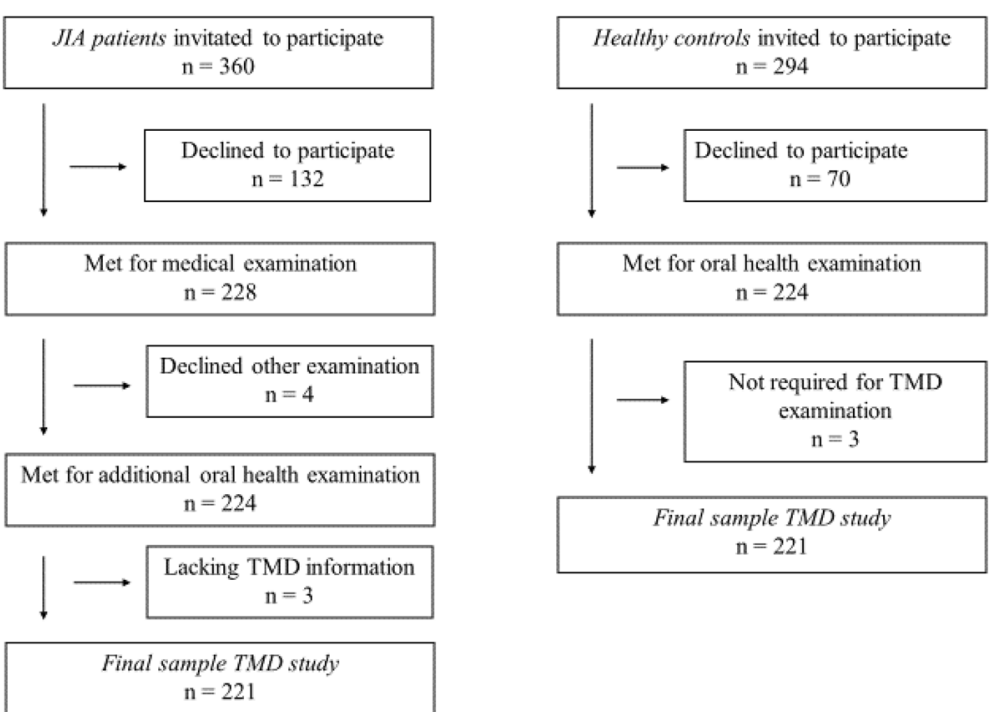

Fig. 1 Flow chart of patients and healthy individuals included in the study

pain outcome, self-reported pain in the jaws during the last 30 days was reported in 59 (26.7\%, 44 girls) participants with JIA vs. 10 (5\%, 8 girls) in healthy controls $(p<0.001)$. Pain during jaw movements at the clinical examination was reported in 112 (51\%, 67 girls) participants with JIA vs. 59 (26.8\%, 34 girls) in healthy controls $(p<0.001)$ (Fig. 2), ranging from 28.6 to $50 \%$ in the different JIA categories (Table 1). No statistically significant differences in the presence of TMD according to JIA categories were found ( $p=0.848)$ (results not shown).

Table 1 Clinical characteristics of participants with Juvenile idiopathic arthritis (JIA) in relation to temporomandibular disorder (TMD)

\begin{tabular}{|c|c|c|c|}
\hline & Total cohort & TMD & No TMD \\
\hline & $n=221$ & $n=88$ & $n=133$ \\
\hline & Value & Value & Value \\
\hline Girls, n (\%) & $132(59.7)$ & $61(69.3)$ & $71(53.4)$ \\
\hline Age at onset, median (IQR) & $6.1(8.1,2.3-10.4)$ & $6.8(8.4,0.7-14.2)$ & $5.2(7.2,0.9-14.7)$ \\
\hline Age at visit by paediatric rheumatologists at the hospital, median (IQR) & $12.7(5.3,9.4-14.7)$ & $13.1(3.3,5.2-16.1)$ & $11.7(6.5,4.8-16.5)$ \\
\hline Disease duration, median (IQR) & $4.6(5.7,2.6-8.3)$ & $4.6(6.0,0.2-14.2)$ & $4.6(5.5,0.2-14.7)$ \\
\hline \multicolumn{4}{|l|}{ JIA categories, n (\%) } \\
\hline Oligoarthritis persistent & $77(34.8)$ & $27(30.7)$ & $50(37.6)$ \\
\hline Oligoarthritis extended & $21(9.5)$ & $11(12.5)$ & $10(7.5)$ \\
\hline Systemic arthritis & $7(3.2)$ & $2(2.3)$ & $5(3.8)$ \\
\hline RF negative polyarthritis & $49(22.2)$ & $17(19.3)$ & $32(24.1)$ \\
\hline RF positive polyarthritis & $4(1.8)$ & $2(2.3)$ & $2(1.5)$ \\
\hline Psoriatic arthritis & $9(4.1)$ & $6(6.8)$ & $3(2.3)$ \\
\hline Enthesitis-related arthritis & $23(10.4)$ & $9(10.2)$ & $14(10.5)$ \\
\hline Undifferentiated JIA & $31(14.0)$ & $14(15.9)$ & $17(12.8)$ \\
\hline \multicolumn{4}{|l|}{ Ongoing medication, n (\%) } \\
\hline No DMARDs & 75 (33.9) & $26(11.8)$ & $49(22.2)$ \\
\hline sDMARDs* & $60(27.1)$ & $23(10.4)$ & $37(16.7)$ \\
\hline bDMARDs** & $86(38.9)$ & 39 (17.6) & $47(21.3)$ \\
\hline
\end{tabular}

JIA Juvenile idiopathic arthritis, TMD Temporomandibular disorder

*sDMARDs = Synthetic disease-modifying antirheumatic- drugs; methotrexate, mykofenolatmofetil, **bDMARDs = Biologic disease-modifying antirheumatic-drugs; etanercept, infliximab, adalimumab, tocilizumab, abatacept, certolizumab, golimumab 


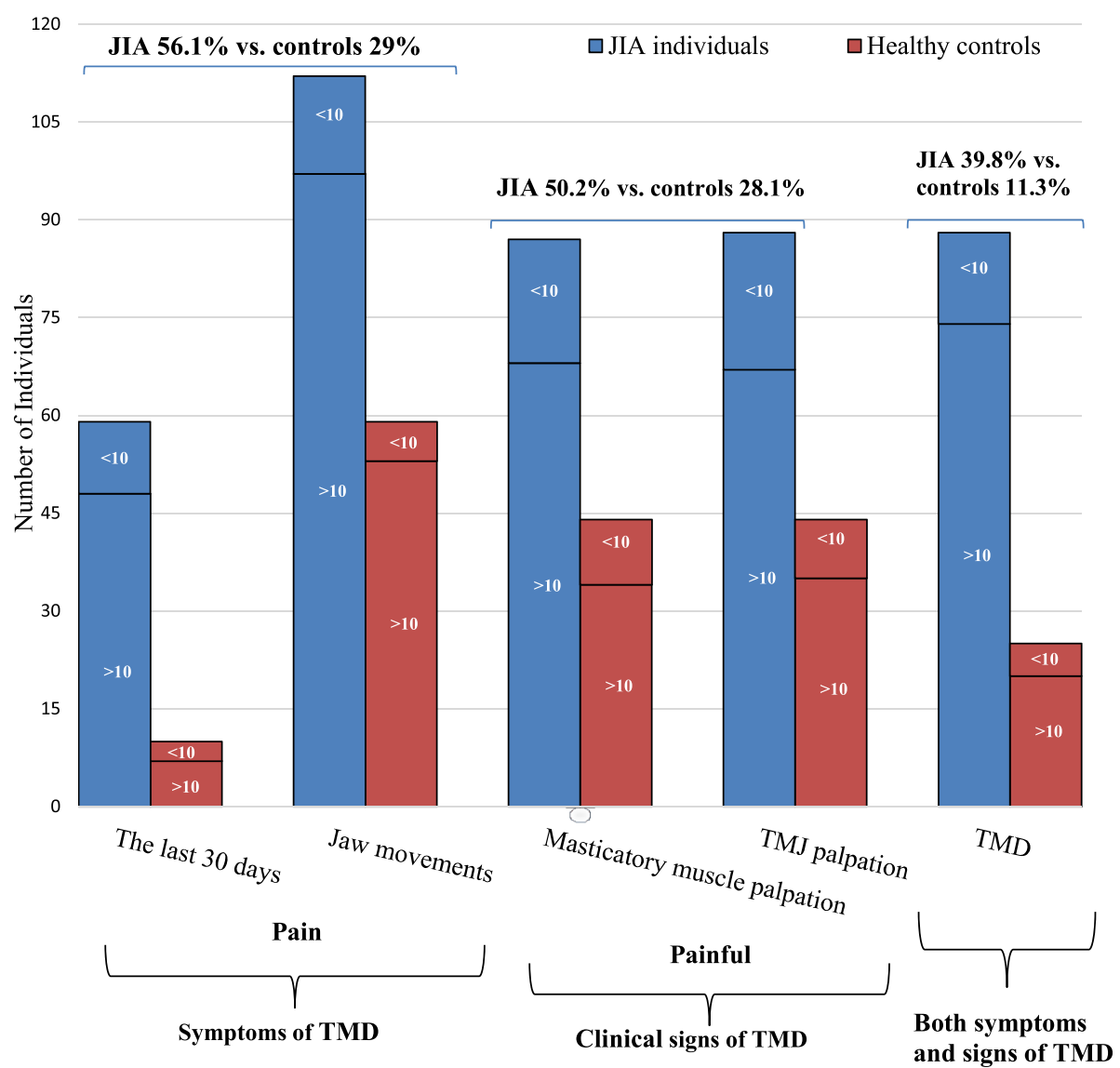

Fig. 2 Prevalence of TMD in children and adolescents with JIA vs. healthy peers, $\geq 10$ years and $<10$ years of age, 1) symptoms: pain the last 30 days and pain with jaw movements; 2) clinical signs: pain upon palpation of the masticatory muscles and TMJ, and 3) a combination of symptoms (1) and clinical signs (2)

The clinical examination revealed that the mean vertical unassisted jaw movement was lower for participants with JIA than for controls, $46.2 \mathrm{~mm}$ vs. $49.0 \mathrm{~mm}$, respectively $(p<0.001)$ (Table 2). A total of 88 (39.8\%, 61 girls) participants with JIA and 25 (11.3\%, 17 girls) healthy controls had both symptoms and clinical signs of TMD (Fig. 2). When assessing the jaw muscles and TM), 111 (50.2\%, 75 girls) participants with JIA had both painful masticatory muscles and TMJs on palpation vs. 62 (28.2\%, 39 girls) of the healthy controls $(\mathrm{p}<0.001)$ (Table 3$)$. A higher proportion of participants on current sDMARDs and/or bDMARDs treatment presented with painful masticatory muscles and TMJ at palpation compared to participants with no biologic treatment (Table 4).

Among participants with JIA, there were no significant differences in vertical unassisted jaw movement according to medication, with a mean of $46.4 \mathrm{~mm}$ (SD 7.1) in the JIA group and $45.8 \mathrm{~mm}$ (SD 7.1) among those not using DMARDs $(p=0.986)$ (results not shown). However, in both groups, more than half of the participants had a vertical unassisted jaw movement of more than 40 $\mathrm{mm}$. The proportion without this medication treatment was slightly higher $(82.7 \%)$ compared to those on current sDMARDs and/or bDMARDs (77.4\%).

Table 2 Jaw movement in 221 participants with JIA compared to controls

\begin{tabular}{|c|c|c|c|c|c|}
\hline Jaw movement & $n$ & $\begin{array}{l}\text { JIA } \\
\text { mm } \\
\text { mean (SD) }\end{array}$ & $n$ & $\begin{array}{l}\text { Healthy controls } \\
\text { mm } \\
\text { mean (SD) }\end{array}$ & $p$-value \\
\hline Vertical unassisted & 221 & $46.2(7.1)$ & 221 & $49.0(6.7)$ & $<0.001$ \\
\hline Lateral to the right side & 215 & $9.7(2.2)$ & 220 & $9.8(2.1)$ & 0.408 \\
\hline Lateral to the left side & 211 & $9.7(2.4)$ & 219 & $10.1(2.0)$ & 0.077 \\
\hline
\end{tabular}


Table 3 Pain on palpation and painful clicking in participants with JIA and controls

\begin{tabular}{|c|c|c|c|c|c|}
\hline Clinical signs & $n$ & $J \mid A, n(\%)$ & $n$ & Healthy controls $n(\%)$ & $p$-value \\
\hline Painful palpation in masticatory muscles \& TMJ & 220 & $111(50.5)$ & 220 & $62(28.2)$ & $<0.001$ \\
\hline Painful palpation in masticatory muscle & 217 & $87(40.1)$ & 218 & $44(20.2)$ & $<0.001$ \\
\hline Painful palpation at the TMJ lateral pole & 220 & $64(29.1)$ & 219 & $29(13.2)$ & $<0.001$ \\
\hline Painful palpation around TMJ lateral pole & 220 & $75(34.1)$ & 219 & $33(15.1)$ & $<0.001$ \\
\hline Painful clicking & 221 & $13(5.9)$ & 221 & $2(0.9)$ & 0.0041 \\
\hline
\end{tabular}

JIA Juvenile idiopathic arthritis, TMJ Temporomandibular joints. ${ }^{*}$ Chi-square test

\section{Discussion}

We have shown using a comparative cross-sectional multicentre design that around one third of the participants with JIA in this cohort had TMD. Half of children and adolescents with JIA reported pain during jaw movements and pain on palpation of the masticatory muscles and TMJs as compared to one fourth of their healthy peers, palpatory pain was associated with sDMARDs and bDMARDs treatment, and children and adolescents with JIA had a significantly lower mean vertical unassisted jaw movement. Moreover, TMJ-related clinical signs and vertical unassisted jaw movement $\leq 40$ $\mathrm{mm}$ had the highest association in the JIA group.

The reported prevalence of TMD in children with JIA varies between 38 and $83 \%$ according to the definitions and methods of ascertainment used, to the cohort examined, and to differences in populations [15, 24-27]. Ferraz and colleagues, in their study of 15 children with JIA ranging in age from 6 to 28 years (mean age 16.3 years), reported a high prevalence of $83 \%$. Still, they did not describe the method of ascertainment, i.e., whether the figures were based on self-reporting or on clinical examination [28]. A previous study from Rongo and colleagues based on 50 participants with JIA aged 9-16 years found a prevalence of TMJ damage from 100 joints to be $74 \%$ as assessed by MRI [25]. Others have reported a prevalence of $55 \%$ based on a questionnaire [29] and of $72 \%$ based on clinical signs [24]. However, none of those studies were based on the research diagnostic criteria RDC/TMD, and the children were older than those in our study. In contrast, a longitudinal study by Zwir et al., including 75 children (mean age 12.4 years), revealed a prevalence of $38 \%$ based on symptoms and $47 \%$ based on clinical examination [30]. Their results are in line with ours.

In our study, the prevalence of TMD, either based on symptoms or clinical signs, in the healthy peers, were quite high at 28 and $29 \%$, respectively. This was higher than in earlier studies among adolescents reported by Graue and colleagues (7 and 12\%, respectively) and Østensjø and colleagues (7\%) [8, 9]. Studies from Finland and Brazil confirm our results with a high prevalence of TMD in the normal population. Vierola et al. [26] reported a TMD prevalence of 35\% (mean age 7.9 years) and de Paiva Bertoli reported a TMD prevalence of $34 \%$ (mean age 11.0 years) [27]. The difference in TMD prevalence in the normal population of children and adolescents is probably due to the use of different diagnostic tools, different numbers of participants, different ages of the studied populations, different countries, and different study designs. In studies from Norway, Graue and colleagues [9] used two screening questions for pain related to TMD [10] and DC/TMD [20] for symptoms and clinical signs in a population of 210 children and adolescents aged 12-19 years. Østensjø et al. [8] used the same two screening questions of TMD symptoms [10] for screening a population of 560 adolescents aged 13-19 years. Then a modified RDC/TMD examination [31] was used for those who answered yes to 1) having pain in the temples, face, TMJ, or jaws once a week or more and 2) having pain once a week or more when opening the mouth wide or chewing. The Finnish group [26] used the RDC/TMD [31] for clinical signs in 483 children aged 6-8 years, and the Brazilian group [27] used

Table 4 Clinical signs and pain at palpation according to DMARDs in 221 participants with JIA

\begin{tabular}{|c|c|c|c|c|c|}
\hline & \multicolumn{2}{|c|}{ Current sDMARDs and/or bDMARDs } & \multicolumn{2}{|c|}{ No current sDMARDs and/or bDMARDs } & \multirow[t]{2}{*}{$p$-value } \\
\hline & $n$ & $\mathrm{n}(\%)$ & $\mathrm{n}$ & n (\%) & \\
\hline Vertical unassisted jaw movement (> 40 mm) & 146 & $113(77.4)$ & 75 & $62(82.7)$ & 0.361 \\
\hline Painful palpation to masticatory muscles \& TMJ & 145 & $80(72.1)$ & 75 & $31(41.3)$ & 0.052 \\
\hline Painful palpation to masticatory muscles & 143 & $62(43.4)$ & 74 & $25(33.8)$ & 0.173 \\
\hline Painful palpation at the TMJ lateral pole & 145 & 49 (33.8) & 75 & $15(20.0)$ & 0.033 \\
\hline Painful palpation at the TMJ around lateral pole & 145 & $56(38.6)$ & 75 & $19(25.3)$ & 0.049 \\
\hline
\end{tabular}

sDMARDs Synthetic disease-modifying antirheumatic-drugs; methotrexate, mykofenolatmofetil, bDMARDs Biologic disease-modifying antirheumatic- drugs; etanercept, infliximab, adalimumab, tocilizumab, abatacept, certolizumab, golimumab, TMJ temporomandibular joint, * Chi-square test 
the American Academy of Orofacial Pain [32] form for screening and the RDC/TMD [31] for clinical examination in a population of 934 individuals aged 10-14 years. Thus it is clear that it can be challenging to get an exact figure on the prevalence of TMD in the normal population. A previous meta-analysis conducted by da Silva and colleagues showed the overall prevalence of intra-articular joint disorder to be $16 \%$ [33].

In our study, approximately half of the JIA subjects had clinical findings consistent with TMD, with no differences according to JIA category. Because the numbers for three of the categories - systemic arthritis, rheumatoid factor positive polyarthritis, and psoriatic arthritis - were relatively low, these results should be interpreted with caution.

The sensitivity and specificity of the clinical orofacial examination in relation to TMJ has been debated because displacement of the disc, although eliciting a clicking sound, might be asymptomatic [34-36]. Based on the DC/TMD criteria, asymptomatic TMJ clicking is still defined as TMD. However, several studies have shown that pain-free clicking represents a normal variant, typically seen in girls during puberty [15]. Recently, a clinical examination protocol for JIA was developed by the Temporomandibular Joint Juvenile Arthritis Working Group (TMJaw). This examination protocol focuses on three general items, namely TMJ symptoms, TMJ dysfunction, and dentofacial deformity in JIA, and it shows acceptable reliability and validity [7].

We found, in accordance with other studies, that the TMJ area and the masseter muscle region were common locations for pain in JIA [29]. However, a recent study from Koos and colleagues reported a lower frequency of masticatory pain on palpation [15], and Kristensen and colleagues stated that masticatory pain complaints could develop over time [37]. In the present study, more than half of the participants with JIA showed clinical signs in the TMJ region and the masseter region, and more than one-fourth of the participants with JIA had TMD. A longitudinal multicentre approach might elucidate the development of masticatory muscle pain, as Kristensen and colleagues have suggested [37].

The vertical unassisted jaw movement has been widely used as a valid marker for TMJ arthritis [38]. We showed that participants with JIA had lower vertical unassisted movements compared to their healthy peers, but the differences were relatively small, thus questioning its clinical significance. Viewed differently, for children and adolescents aged $<11$ years, the cut-off value of $40 \mathrm{~mm}$ was within the range of normal vertical jaw movement [39]. Further, our findings suggest that lateral movement did not differ significantly between the two groups, which is in line with the results of Twilt and colleagues [40] and Küseler and colleagues [19]. In the latter study of 15 children with JIA with a mean age of 12 years, the recorded decreased lateral movements were $\leq 5 \mathrm{~mm}$ with no significant relevance [19].

We found no statistically significant differences in the presence of TMD according to JIA categories. However, we found a significantly higher occurrence of clinical signs in participants with JIA currently on DMARDs medication (whether synthetic or biologic) compared to those not taking such medication. A high risk of developing clinical signs of TMD was associated with a severe disease course, as indicated by the use of DMARDs.

The strengths of this study are the relatively large number of participants, in which the study groups were well matched, and the meticulous standardisation of the clinical TMJ assessment performed prior to and during the study period. However, the large number of participants should not hide the fact that we are dealing with an underpowered sample size that was lacking 75 participants. An additional limitation is that the overall response rate of $63 \%$, although considered acceptable, might have influenced the results because the group that did not participate was, on average, slightly younger and had a somewhat lower proportion of girls. Also, the shortened version of the DC/TMD used in this study is not directly comparable with studies having used the full DC/TMD score. In the present study, children and adolescents with JIA with TMD involvement were defined based on clinical examination, and both self-reported and parent-reported pain. Further studies will focus on the role of imaging on the diagnosis of TMJ arthritis in children and adolescents with JIA. Clinical orofacial examination may not be reliable for diagnosing disc displacement without reduction [5]. Imaging diagnosis is particularly important in JIA with non-symptomatic TMJ involvement because hard tissue loss in the condyle might hinder the growth of the mandible and subsequently affect chewing function and cause aesthetic problems [15].

\section{Conclusion}

Symptoms or clinical signs of TMD were seen in approximately half of the participants with JIA compared to about one fourth of their healthy peers. Painful palpation of masticatory muscles and decreased vertical unassisted jaw movement are more frequent in children with JIA than in healthy controls and should be part of both medical and dental routine examinations in the follow-up of JIA.

\section{Supplementary information}

Supplementary information accompanies this paper at https://doi.org/10. 1186/s12903-020-01234-z.

Additional file 1 Table S1. Reliability tests (using intraclass correlation coefficients) between "a reference" and the examiners. 
Additional file $\mathbf{2}$ Table S2. Percent agreement values between "a reference" and the examiners.

\section{Abbreviations}

DC/TMD: Diagnostic criteria for temporomandibular disorder; bDMARDs: Biological disease-modifying antirheumatic drugs; DMAR Ds: Disease-modifying antirheumatic drugs; sDMARDs: Synthetic diseasemodifying antirheumatic drugs; ILAR: International League of Associations of Rheumatology; JIA: Juvenile idiopathic arthritis; RDC/TMD: Research diagnostic criteria for TMD; TMD: Temporomandibular disorder; TMJ: Temporomandibular joint

\section{Acknowledgements}

The authors thank Prof. Ellen Berit Nordal for her constructive comments on the manuscript. We thank the Center for Oral Health Services and Research of Middle-Norway (TkMidt), Trondheim, the Public Dental Service Competence Centre of Northern-Norway (TkNN), Troms $\varnothing$ and the Oral Health Centre of Expertise in Western Norway (TkVest), Bergen, for contributing with time and personnel resources. Thanks and appreciation to Marie Sager and Gunnar Lyngstad Center for Oral Health Services and Research of Middle-Norway (TkMidt). We thank the entire NorJIA research group (NorJIA.com) and Kasper Dahl Kristensen, Section of Orthodontics, Aarhus University, Denmark, for help with the questionnaires. Our gratitude also goes to all the participants accepting to be included in the study.

\section{Authors' contributions}

JF: contributed to the conception and design of the study, agreed to be accountable for all aspects of the work and ensure questions related to the accuracy or integrity of any part of the work were appropriately investigated and resolved, was involved in writing the manuscript, and approved the final version to be published. MSS, KR, XQS, MR, PS, AR: contributed to the conception and design of the study and analysis/ interpretation of the data, were involved in drafting the manuscript and revising it critically for important intellectual content, and approved the final version to be published. SL: performed biostatistics. KT, EGG, LC, JH, LVWM, PF: contributed to data collection and provided valuable comments. All authors have read and approved the final manuscript.

\section{Funding}

The current study is supported in part by the Norsk Revmatikerforbund (Norway).

\section{Availability of data and materials}

The datasets used and analysed in the current study are available from the corresponding author on reasonable request.

\section{Ethics approval and consent to participate}

The study was approved by the regional ethics committee (2012/542/REK vest). Written informed consents were obtained from the parents/legal representatives and the adolescents. The study was registered at ClinicalTrials.gov (No: NCT03904459).

\section{Consent for publication}

Not applicable.

\section{Competing interests}

The authors declare that they have no competing interests.

\section{Author details}

${ }^{1}$ Department of Clinical Dentistry, The Faculty of Medicine, University of Bergen, Årstadveien 19, N-5009 Bergen, Norway. ${ }^{2}$ Center for Oral Health Services and Research of Middle-Norway (TkMidt), Trondheim, Norway. ${ }^{3}$ Department of Radiology, University Hospital of North Norway, Troms $\varnothing$, Norway. ${ }^{4}$ UiT the Arctic University of North Norway, Tromsø, Norway. ${ }^{5}$ Department of Pediatrics, Haukeland University Hospital, Bergen, Norway. ${ }^{6}$ Department of Oral and Maxillofacial Radiology, Faculty of Odontology, University of Malmö, Malmö, Sweden. ${ }^{7}$ Public Dental Service Competence Centre of Northern-Norway (TkNN), Troms $\varnothing$, Norway. ${ }^{8}$ Department of Clinical and Molecular Medicine, NTNU - Norwegian University of Science and Technology, Trondheim, Norway. ${ }^{9}$ Department of Pediatrics, St. Olavs
Hospital, Trondheim, Norway. ${ }^{10}$ Department of Otorhinolaryngology, Division of Oral and Maxillofacial Surgery, University Hospital North Norway, Tromsø, Norway. ${ }^{11}$ Department of Clinical Medicine, Faculty of Health Sciences, The Arctic University of Norway, Tromsø, Norway. ${ }^{12}$ Section of Orthodontics, Faculty of Health Sciences, Aarhus University, Aarhus, Denmark.

${ }^{13}$ Department of Oral and Maxillofacial Surgery, Haukeland University Hospital, Bergen, Norway.

Received: 29 June 2020 Accepted: 25 August 2020

Published online: 13 October 2020

\section{References}

1. Riise OR, Handeland KS, Cvancarova M, Wathne K-O, Nakstad B, Abrahamsen $\mathrm{TG}$, et al. Incidence and characteristics of arthritis in Norwegian children: a population-based study. Pediatrics. 2008.

2. Gabriel SE, Michaud K. Epidemiological studies in incidence, prevalence, mortality, and comorbidity of the rheumatic diseases. Arthritis Res Ther. 2009;11:229. https://doi.org/10.1186/ar2669.

3. Petty RE, Southwood TR, Manners P, Baum J, Glass DN, Goldenberg J, et al. International league of associations for rheumatology classification of juvenile idiopathic arthritis: second revision, Edmonton, 2001. J Rheumatol. 2004:31:390-2 PMID: 14760812

4. LeResche L. Epidemiology of temporomandibular disorders: implications for the investigation of etiologic factors. Crit Rev Oral Biol Med. 1997.

5. Peck CC, Goulet JP, Lobbezoo F, Schiffman EL, Alstergren P, Anderson GC, et al. Expanding the taxonomy of the diagnostic criteria for temporomandibular disorders. J Oral Rehabil. 2014;41(1):2.

6. Ohrbach R, Dworkin SF. The Evolution of TMD Diagnosis. J Dent Res. 2016: 0022034516653922. https://doi.org/10.1177/0022034516653922 PMID: 27313164.

7. Stoustrup P, Herlin T, Spiegel L, Rahimi H, Koos B, Pedersen TK, et al. Standardizing the clinical orofacial examination in Juvenile idiopathic arthritis: An interdisciplinary, consensus-based, short screening protocol. J Rheumatol. 2019;190661 jrheum.

8. Ostensjo V, Moen K Storesund T, Rosen A. Prevalence of painful Temporomandibular disorders and correlation to lifestyle factors among adolescents in Norway. Pain Res Manag. 2017;2017:2164825. https://doi.org/ $10.1155 / 2017 / 2164825$

9. Graue AM, Jokstad A, Assmus J, Skeie MS. Prevalence among adolescents in Bergen, Western Norway, of temporomandibular disorders according to the DC/TMD criteria and examination protocol. Acta Odontol Scand. 2016;74: 449-55.

10. Nilsson IM, List T, Drangsholt M. Prevalence of temporomandibular pain and subsequent dental treatment in swedish adolescents. J Orofac Pain. 2005. 15895837

11. Cannizzaro E, Schroeder S, Müller LM, Kellenberger CJ, Saurenmann RK. Temporomandibular joint involvement in children with juvenile idiopathic arthritis. J Rheumatol. 2011:38:510-5. https://doi.org/10.3899/jrheum.100325.

12. Stoustrup $P$, Glerup $M$, Bilgrau $A E$, Küseler $A$, Verna $C$, Christensen $A E$, et al. Cumulative incidence of Orofacial manifestations in early juvenile idiopathic arthritis: a regional, Three-Year Cohort Study. Arthritis Care Res (Hoboken). 2020;72(7):907.

13. Küseler A, Pedersen TK, Herlin T, Gelineck J. Contrast enhanced magnetic resonance imaging as a method to diagnose early inflammatory changes in the temporomandibular joint in children with juvenile chronic arthritis. J Rheumatol. 1998;25:1406-12

14. Billiau AD, Hu Y, Verdonck A, Carels C, Wouters C. Temporomandibular joint arthritis in juvenile idiopathic arthritis: prevalence, clinical and radiological signs, and relation to Dentofacial morphology. J Rheumatol. 2007: PMID: 17696265 .

15. Koos B, Twilt M, Kyank U, Fischer-Brandies H, Gassling V, Tzaribachev N. Reliability of clinical symptoms in diagnosing temporomandibular joint arthritis in juvenile idiopathic arthritis. J Rheumatol. 2014;41:1871-7. https:// doi.org/10.3899/jrheum.131337.

16. Stoll ML, Sharpe T, Beukelman T, Good J, Young D, Cron RQ. Risk factors for temporomandibular joint arthritis in children with juvenile idiopathic arthritis. J Rheumatol. 2012;39:1880-7. https://doi.org/10.3899/jrheum. 111441

17. Keller H, Müller LM, Markic G, Schraner T, Kellenberger CJ, Saurenmann RK. Is early TMJ involvement in children with juvenile idiopathic arthritis clinically detectable? Clinical examination of the TMJ in comparison with 
contrast enhanced MRI in patients with juvenile idiopathic arthritis. Pediatr Rheumatol. 2015;13:56. https://doi.org/10.1186/s12969-015-0056-2.

18. Weiss PF, Arabshahi B, Johnson A, Bilaniuk LT, Zarnow D, Cahill AM, et al. High prevalence of temporomandibular joint arthritis at disease onset in children with juvenile idiopathic arthritis, as detected by magnetic resonance imaging but not by ultrasound. Arthritis Rheum. 2008;58:118996. https://doi.org/10.1002/art.23401.

19. Küseler A, Pedersen TK, Gelineck J, Herlin T. A 2 year followup study of enhanced magnetic resonance imaging and clinical examination of the temporomandibular joint in children with juvenile idiopathic arthritis. J Rheumatol. 2005; PMID: 15630742.

20. Schiffman E, Ohrbach R, Truelove E, Look J, Anderson G, Goulet J-P, et al. Diagnostic Criteria for Temporomandibular Disorders (DC/TMD) for Clinical and Research Applications: Recommendations of the International RDC/ TMD Consortium Network* and Orofacial Pain Special Interest Groupt. J Oral Facial Pain Headache. 2014;28:6-27. https://doi.org/10.11607/jop.1151.

21. Stoustrup P, Koos B. Clinical craniofacial examination of patients with juvenile idiopathic arthritis. Semin Orthod. 2015;21:94-101.

22. Skeie MS, Frid P, Mustafa M, Aßmus J, Rosén A. DC/TMD examiner protocol: longitudinal evaluation on interexaminer reliability. Pain Res Manag. 2018; 2018.

23. Svensson B, Adell R, Kopp S. Temporomandibular disorders in juvenile chronic arthritis patients. A clinical study. Swed Dent J. 2000;24:83-92. 11 061206

24. Leksell E, Ernberg M, Magnusson B, Hedenberg-Magnusson B. Orofacial pain and dysfunction in children with juvenile idiopathic arthritis: a case-control study. Scand J Rheumatol. 2012.

25. Rongo R, Alstergren P, Ammendola L, Bucci R, Alessio M, D'Antò V, et al. Temporomandibular joint damage in juvenile idiopathic arthritis: diagnostic validity of diagnostic criteria for temporomandibular disorders. J Oral Rehabil. 2019;46(5):450

26. Vierola A, Suominen AL, Ikavalko T, Lintu N, Lindi V, Lakka H-M, et al. Clinical signs of temporomandibular disorders and various pain conditions among children 6 to 8 years of age: the PANIC study. J Orofac Pain. 2012. 22292136

27. de Paiva Bertoli FM, Bruzamolin CD, Pizzatto E, Losso EM, Brancher JA, de Souza JF. Prevalence of diagnosed temporomandibular disorders: a crosssectional study in Brazilian adolescents. PLoS One. 2018;13. 29420573.

28. Ferraz AML, Devito KL, Guimarães JP. Temporomandibular disorder in patients with juvenile idiopathic arthritis: clinical evaluation and correlation with the findings of cone beam computed tomography. Oral Surg Oral Med Oral Pathol Oral Radiol. 2012;114:e51-7. https://doi.org/10.1016/j.00oo. 2012.02.010

29. Rahimi H, Twilt M, Herlin T, Spiegel L, Pedersen TK, Küseler A, et al. Orofacial symptoms and oral health-related quality of life in juvenile idiopathic arthritis: a two-year prospective observational study. Pediatr Rheumatol. 2018;16.

30. Zwir LMLF, Terreri MTRA, Sousa SA, Fernandes ARC, Guimarães AS, Hilário MOE. Are temporomandibular joint signs and symptoms associated with magnetic resonance imaging findings in juvenile idiopathic arthritis patients? A longitudinal study. Clin Rheumatol. 2015;34:2057-63.

31. Dworkin SF, LeResche L. Research diagnostic criteria for temporomandibular disorders: review, criteria, examinations and specifications, critique. J Craniomandib Disord. 1992:6:301-55. 1298767.

32. de Leeuw RRJ, Klasser G. American Academy of Orofacial Pain. General assessment of the orofacial pain patient. Orofacial pain - guidelines for assessment, diagnosis and management. 5th ed. Chicago: Quintessence; 2013. In: Orofacial pain; guidelines for assessment, diagnosis, and management, 5th ed. Quintessence Pub. Co.; 2013. p. 25-46. https://searchproquest-com.pva.uib.no/docview/1416243368?accountid $=8579$.

33. Da Silva CG, Pachêco-Pereira C, Porporatti AL, Savi MG, Peres MA, Flores-Mir $C$, et al. Prevalence of clinical signs of intra-articular temporomandibular disorders in children and adolescents A systematic review and metaanalysis. J Am Dental Association. 2016;147(1):10

34. Kircos LT, Ortendahl DA, Mark AS, Arakawa M. Magnetic resonance imaging of the TMJ disc in asymptomatic volunteers. J Oral Maxillofac Surg. 1987; 45(10):852.

35. Westesson PL, Eriksson L, Kurita K. Reliability of a negative clinical temporomandibular joint examination: prevalence of disk displacement in asymptomatic temporomandibular joints. Oral Surgery, Oral Med Oral Pathol. 1989.
36. Isberg A, Stenström B, Isacsson G. Frequency of bilateral temporomandibular joint disc displacement in patients with unilateral symptoms: a 5-year follow-up of the asymptomatic joint. A clinical and arthrotomographic study. Dentomaxillofacial Radiol. 1991;20(2):73.

37. Kristensen KD, Stoustrup P, Kuseler A, Pedersen TK, Twilt M, Herlin T. Clinical predictors of temporomandibular joint arthritis in juvenile idiopathic arthritis: a systematic literature review. Semin Arthritis Rheum. 2016;45:71732. https://doi.org/10.1016/j.semarthrit.2015.11.006.

38. Stoustrup P, Kristensen KD, Verna C, Küseler A, Herlin T, Pedersen TK. Orofacial symptoms related to temporomandibular joint arthritis in juvenile idiopathic arthritis: smallest detectable difference in self-reported pain intensity. J Rheumatol. 2012;39:2352-8.

39. Stoustrup P, Kristensen KD, Küseler A, Herlin T, Pedersen TK. Normative values for mandibular mobility in Scandinavian individuals 4-17 years of age. J Oral Rehabil. 2016;43:591-7. https://doi.org/10.1111/joor.12407.

40. Twilt M, Mobers SMLM, Arends LR, Ten Cate R, Van Suijlekom-Smit LWA Temporomandibular involvement in juvenile idiopathic arthritis. J Rheumatol. 2004;31:1418-22. 15229966.

\section{Publisher's Note}

Springer Nature remains neutral with regard to jurisdictional claims in published maps and institutional affiliations.
Ready to submit your research? Choose BMC and benefit from:

- fast, convenient online submission

- thorough peer review by experienced researchers in your field

- rapid publication on acceptance

- support for research data, including large and complex data types

- gold Open Access which fosters wider collaboration and increased citations

- maximum visibility for your research: over $100 \mathrm{M}$ website views per year

At BMC, research is always in progress.

Learn more biomedcentral.com/submissions 